\title{
Forecasting of Taiwan's Gross Domestic Product using the Novel Nonlinear Grey Bernoulli Model with ANN Error Correction
}

\section{Chen $\mathrm{Cl}^{1 *}$ and $\mathrm{Hsin} \mathrm{PH}^{2}$}

${ }^{1}$ Deaprtment of Industrial Management, I-Shou University, Taiwan

${ }^{2}$ Department of International Business, Cheng Shiu University, Taiwan

\begin{abstract}
Nonlinear Grey Bernoulli Model is proposed to enhance the prediction accuracy. In this study, artificial neural network (ANN) is used to modify the residual error of NGBM. Then, ANN error plus original forecasted value is a new estimated value. The newly proposed method termed NGBM $(1,1)$ with ANN error correction is used to forecast Taiwan's gross domestic product (GDP). The results show the proposed method is more accurate than NGBM and is proven to be effective in forecasting.
\end{abstract}

Keywords: Grey forecasting; Artificial neural network; Gross domestic product; Nonlinear Grey Bernoulli Model

\section{Introduction}

Grey theory [1] has been proposed over 30 years. Great endeavor has been devoted to increase the forecasting precision. One of methods treated the forecasting error to become the modified grey forecasting model. Hsu and Wen [2] modified original GM $(1,1)$ models are improved by using residual modifications with Markov chain sign estimations. Hsu and Chen [3] improved grey GM $(1,1)$ model, using a technique that combines residual modification with artificial neural network sign estimation, is proposed. Hsu [4] applied three residual modification models to enhance the forecasting results. The result showed the Markov-chain residual modification model achieves reliable and precise results. Zhou et al. [5] presented a trigonometric grey prediction approach by combining the traditional grey model GM $(1,1)$ with the trigonometric residual modification technique for forecasting electricity demand. Jing [6] applied residual grey model $(1,1)$ to the prediction of tuberculosis prevalence.

Above residual modification is based on linear GM $(1,1)$ model. Recently, a nonlinear grey forecasting model which was termed NGBM was proposed. Chen et al. [7] proposed NGBM to forecast the foreign exchange rates of Taiwan's major trading partners by novel nonlinear Grey Bernoulli model NGBM. Then, an improved NGBM model [8] was proposed to further increase the forecasting precision. Furthermore, Wang [9] used an optimized Nash nonlinear grey Bernoulli model for forecasting the main economic indices of high technology enterprises in China. Zhang [10] used a particle swarm optimization algorithm to solve the optimal parameter estimation problem, an improved Nash nonlinear grey Bernoulli model termed PSO-NNGBM $(1,1)$ is proposed.

Through literature review, the NGBM with residual correction model has not been proposed. This study connects artificial neural network (ANN) with the Nonlinear Grey Bernoulli Model (NGBM) to develop a new hybrid Grey model, named NGBM with ANN error correction.

Gross domestic product (GDP) is the market value of all final goods and services produced within a country in a given period. It is often considered an indicator of a country's standard of living. Besides, GDP is a sum of consumption, investment, government spending and net exports. Therefore, the consumers, the investors, the firms and the policy makers all care about the indicator of GDP. In this study, the proposed model is used to forecast Taiwan's GDP. In testing period for 2011, the results indicate that the proposed model has a small estimated error. Besides, this study uses this method to forecast Taiwan's GDP for 2012. The results show Taiwan is away from financial crisis and on the road to prosperity.

This paper is organized as follows. Section 2 introduces the mathematics of the Nonlinear Grey Bernoulli Model with ANN error correction, and defines the forecasting relative percentage error. Section 3 applies the proposed method to forecasting Taiwan's GDP. Finally, Section 4 presents conclusions.

\section{Methodology}

The derivation of linear GM $(1,1)$ and nonlinear NGBM are briefly introduced in the section. In order to increase the forecasting precision, the artificial neural network (ANN) is incorporated in the NGBM error correction model. The concept of ANN is also demonstrated.

\section{The nonlinear grey Bernoulli model with ANN error correction}

Step 1: Cpllect the original series of data with $m$ entries is:

$$
X^{(0)}(1, m)=\left\{x^{(0)}(1), x^{(0)}(2), \ldots, x^{(0)}(k), . ., x^{(0)}(m)\right\},
$$

where raw matrix $X^{(0)}$ represents the non-negative original time series data.

Step 2: Construct $X^{(1)}$ using a one time accumulated generation operation (1-AGO), namely

$$
X^{(1)}(1, m)=\left\{x^{(1)}(1), \ldots, x^{(1)}(k), \ldots, x^{(1)}(m)\right\},
$$

Where,

${ }^{*}$ Corresponding author: Chen $\mathrm{Cl}$, Deaprtment of Industrial Management, I-Shou University, Taiwan, Tel: +886 7 6577711; E-mail: eddychen777@gmail.com

Received December 11, 2015; Accepted February 09, 2016; Published February 12,2016

Citation: Chen $\mathrm{Cl}$, Hsin PH (2016) Forecasting of Taiwan's Gross Domestic Product using the Novel Nonlinear Grey Bernoulli Model with ANN Error Correction. J Glob Econ 4: 177. doi:10.4172/2375-4389.1000177

Copyright: $\odot 2016 \mathrm{Chen} \mathrm{Cl}$, et al. This is an open-access article distributed unde the terms of the Creative Commons Attribution License, which permits unrestricted use, distribution, and reproduction in any medium, provided the original author and source are credited. 


$$
x^{(1)}(k)=\sum_{i=1}^{k} x^{(0)}(i), k=1,2, \ldots, m
$$

Step 3: 1-AGO yields a monotonically increasing sequence similar to the solution curve of the first order linear differential equation. Therefore, the solution curve of the following differential equation approximates the 1-AGO data.

$$
\frac{d \hat{x}^{(1)}}{d t}+\alpha \hat{x}^{(1)}=\beta
$$

Where, $\wedge$ represents the Grey predicted value. The $\alpha$ and $\beta$ are model parameters; $\hat{x}^{(1)}(1)=x^{(0)}(1)$ is the initial condition.

Step 4: Model parameters $\alpha$ and $\beta$ can be calculated by discretization of Eq. (4)

$$
\frac{d \hat{x}^{(1)}}{d t}=\lim _{\Delta t \rightarrow 0} \frac{\hat{x}^{(1)}(t+\Delta t)-\hat{x}^{(1)}(t)}{\Delta t}
$$

let $\Delta t \rightarrow 1$, in which case 1-AGO approximates the forecast value,

$$
\frac{d \hat{x}^{(1)}}{d t} \cong x^{(1)}(k+1)-x^{(1)}(k)=x^{(0)}(k+1), k=1,2,3, \ldots, m
$$

$X^{(1)}$, the background value, is defined as

$$
\hat{x}^{(1)}(t) \cong p x^{(1)}(k)+(1-p) x^{(1)}(k+1)=z^{(1)}(k+1), k=1,2,3, \ldots
$$

Where, the production coefficient $p$ is in the range $0-1$, and traditionally equals 0.5 . Thus, the source model can be obtained as follows:

$$
x^{(0)}(k)+\alpha z^{(1)}(k)=\beta, k=1,2, \ldots, m
$$

The model parameters $\alpha$ and $\beta$ can be solved by least square methods.

$$
\left[\begin{array}{l}
\alpha \\
\beta
\end{array}\right]=\left(Z^{T} Z\right)^{-1} Z^{T} Y
$$

where $Z$ and $Y$ are defined as follows:

$$
\mathrm{Z}=\left[\begin{array}{cc}
z^{(1)}(2) & 1 \\
z^{(1)}(3) & 1 \\
\vdots & \vdots \\
z^{(1)}(m) & 1
\end{array}\right]=\left[\begin{array}{c}
x^{(0)}(2) \\
x^{(0)}(3) \\
\vdots \\
x^{(0)}(m)
\end{array}\right]
$$

Step 5: Solve Eq. (4) together with the initial condition, and the particular solution is

$$
\hat{x}^{(1)}(k+1)=\left(x^{(0)}(1)-\frac{\beta}{\alpha}\right) e^{-a k}+\frac{\beta}{\alpha}, k=1,2, \ldots, m
$$

Consequently, the prediction output at step $k$ can be estimated using the inverse accumulated generating operation (IAGO). It is defined as

$$
\hat{x}^{(1)}(k+1)-\hat{x}^{(1)}(k)
$$

or

$$
\hat{x}^{(0)}(k+1)=\left(1-e^{-\alpha}\right)\left(x^{0}(1)-\frac{\beta}{\alpha}\right) e^{-\alpha k}, k=1,2,, \ldots, m
$$

Step 6: Equation (4) is linear differential equation and the only adjustable variable is background value $p$. Based on the elementary course in ordinary differential equation, a similar form of differential equation to eq. (4) is called Bernoulli equation [11], which is nonlinear and has the following form,

$$
\frac{d \hat{x}^{(1)}}{d t}+\alpha \hat{x}^{(1)}=\beta\left[\hat{x}^{(1)}\right]^{n}
$$

Where, $n$ belongs to any real number except one. There are two special cases of NGBM. When $n=0$, the equation is original grey forecasting model. When $n=2$, the equation is Grey-Verhulst equation [12].

Step 7: A discrete form of eq. (14) is described as.

$$
x^{(0)}(k)+\alpha z^{(1)}(k)=\beta\left[z^{(1)}(k)\right]^{n}, k=2,3,4, \ldots
$$

By least square method, the above model parameters $a$ and $b$ become

$$
\left[\begin{array}{l}
\alpha \\
\beta
\end{array}\right]=\left(B^{T} B\right)^{-1} B^{T} Y
$$

where $B$ and $Y$ are defined as follows

$$
B=\left[\begin{array}{cc}
-z^{(1)}(2) & {\left[z^{(1)}(2)\right]^{n}} \\
-z^{(1)}(3) & {\left[z^{(1)}(3)\right]^{n}} \\
\vdots & \vdots \\
-z^{(1)}(m) & {\left[z^{(1)}(m)\right]^{n}}
\end{array}\right], Y=\left[\begin{array}{c}
x^{(0)}(2) \\
x^{(0)}(3) \\
\vdots \\
x^{(0)}(m)
\end{array}\right]
$$

Step 8: The corresponding particular solution of eq. (14) is

$$
\hat{x}^{(1)}(k+1)=\left[\left(x^{(0)}(1)^{(1-n)}-\frac{\beta}{\alpha}\right) e^{-a(1-n) k}+\frac{\beta}{\alpha}\right]^{1 /(1-n)}, n \neq 1, k=1,2,3, \ldots
$$

Step 9: Take the inverse accumulated generation operation (IAGO) on $\hat{x}^{(1)}(k+1)$ to obtain the estimated value $\hat{x}^{(0)}(k)$. IAGO is defined in eq (12).

The error between forecasting and raw data is inevitable; therefore, the minimization of error is one approach to increase forecasting precision. By the conception of error correction, this study proposes a novel grey model, named NGBM with ANN error correction. The original forecast value is obtained by NGBM; the estimated forecast error is calculated by ANN. Finally, the original forecast value plus the estimated forecast error is the proposed forecast value. The procedure for obtaining ANN error correction is described as follows.

Step 10: One of modified residual GM $(1,1)$ model was proposed by Deng [1]. In this research, the ANN residual model is proposed. The differences between the real

values, $x^{(0)}(k)$, and the forecasted values, $\hat{x}^{(0)}(k)$, are defined as the residual series. The residual series is

$$
\left[\begin{array}{l}
\alpha \\
\beta
\end{array}\right]=\left(B^{T} B\right)^{-1} B^{T} Y E^{(0)}(1, m)=\left\{e^{(0)}(1), e^{(0)}(2), \ldots, e^{(0)}(k), . ., e^{(0)}(m)\right\},
$$

where $e^{(0)}(k)=x^{0}(k)-\hat{x}^{0}(k)$

\section{Step 11: Let}

$\varepsilon^{(0)}(k)=\left[e^{0}(k)-\operatorname{Min}\left\{E^{(0)}(1, m)\right\}\right] /\left[\operatorname{Max}\left\{E^{(0)}(1, m)\right\}-\operatorname{Min}\left\{E^{(0)}(1, m)\right\}\right]$

Where

$$
\varepsilon^{(0)}(k)=\left[e^{0}(k)-\operatorname{Min}\left\{E^{(0)}(1, m)\right\}\right] /\left[\operatorname{Max}\left\{E^{(0)}(1, m)\right\}-\operatorname{Min}\left\{E^{(0)}(1, m)\right\}\right]
$$

Note that $\varepsilon^{(0)}(k)$ is a standardized error.

Step 12: Use $E_{S}^{(0)}(k, k+3)$ to forecast $\varepsilon^{(0)}(k+4)$ by ANN. The parameters about ANN are described in section 3 .

Step 13: Thus, the forecasted values is defined as $\hat{\varepsilon}_{A N N}{ }^{(0)}(k+4)$ 
Step 14: Calculate the forecasting value with ANN error correction. Here, it is

$$
\varepsilon^{(0)}(k)=\left[e^{0}(k)-\operatorname{Min}\left\{E^{(0)}(1, m)\right\}\right] /\left[\operatorname{Max}\left\{E^{(0)}(1, m)\right\}-\operatorname{Min}\left\{E^{(0)}(1, m)\right\}\right] \hat{x}_{A N N}{ }^{(0)}(k+1)
$$$$
=\hat{x}^{(0)}(k+1)+\left\{\left(\hat{\varepsilon}_{A N N}{ }^{(0)}(k+1) *\left[\operatorname{Max}\left\{E^{(0)}(k)\right\}-\operatorname{Min}\left\{E^{(0)}(k)\right\}\right]\right)-\operatorname{Min}\left\{E^{(0)}(k)\right\}\right\}
$$

\section{Measures of forecasting performance}

The residual error test is the main criterion for assessing prediction accuracy. Grey forecasting usually uses (average) relative percentage error to examine the forecast precision. Generally speaking, the model which has small residual error is regard as a good model. The relative percentage error $(R P E)$ and the average relative percentage error $(A R P E)$ are defined as

$$
\begin{aligned}
& R P E=\frac{x^{(0)}\left(t_{i}\right)-\hat{x}^{(0)}\left(t_{i}\right)}{x^{(0)}\left(t_{i}\right)} \times 100 \% \\
& =\zeta(Z, Y(n)) \\
& A R P E=\frac{1}{m-1} \sum_{i=1}^{m}|\zeta(B, Y(n))| \\
& =\psi[\zeta(Z, Y(n))]
\end{aligned}
$$

\section{Forecasting Taiwan's GDP}

In this study, Taiwan's GDP is used to verify the proposed model. Data is from Ministry of Economic Affairs [13] in Taiwan. GDP is the market value of all of the final goods and services produced within the country's borders in a one-year period. GDP can be determined in three ways which are the product approach, the income approach, and the expenditure approach. In principle, they have the same result. The expenditure approach method is broadly used and easy to calculate. GDP (Y) is a sum of consumption (C), investment (I), government spending $(G)$ and net exports $(X-M)$. That is, $Y=C+I+G+(X$ $-\mathrm{M})$. C (consumption) is usually the largest GDP component in the economy, consisting of household final consumption expenditure in the economy. I (investment) contain business investment in fixtures and equipment, but do not include exchanges of existing assets. G (government spending) is the sum of government expenditures on final goods and services. It comprises salaries of public servants, purchase of weapons for the military, and any investment expenditure by a government. X (exports) expresses gross exports. GDP measures the amount a country produces, including goods and services produced for other nations' consumption, therefore exports are added. M (imports) expresses gross imports. Imports are subtracted since imported goods will be included in the terms $\mathrm{G}$, I, or $\mathrm{C}$, and must be deducted to avoid counting foreign supply as domestic.

Data from 2004 to 2010 is used to construct the proposed model. And then forecast Taiwan's GDP for 2011 to verify the proposed model. Every series containing four data is regard as inputs and the firth data is output.

\section{Empirical results of NGBM $(1,1)$}

The process of obtaining the forecasted values by NGBM $(1,1)$ is described in the following.

(1) Denote the original data series,

$X^{(0)}(1,7)=\{339973,364832, \ldots, 430149\}$,

(2) Thus, the AGO series of $\{522389,892992.5, \ldots, 2467049.5\}$,

(3) And, the background value series are
$\{522389,892992.5, \ldots, 2467049.5\}$,

(4) After constructing the matrices $Z$ and $Y$, solve the following objective function.

$$
\begin{aligned}
\underset{\operatorname{Min}_{\{n\}} \text { ARPE }}{\sin } & =\frac{1}{m-1} \sum_{2}^{m}\left(\frac{\left|x^{(0)}\left(t_{i}\right)-\hat{x}^{(0)}\left(t_{i}\right)\right|}{x^{(0)}\left(t_{i}\right)} \times 100 \%\right) \\
& =\psi[\zeta(Z, Y(n))] \\
& =\psi[n]
\end{aligned}
$$

By Newtonian method, $n$ is 0.03 and $A R P E$ is 2.3289

(5) The estimated value series by NGBM are listed in Table 1.

(6) For forecasting GDP of 2012, the data of 2011 is treated as new information. So, the new original data series is $E^{(0)}(1,7)=\{0,467.12, \ldots, 44757.57\}$, After repeating above procedure, the estimated value of $2012, X^{(0)}(9)$, is $430647.04(\mathrm{n}=-0.06$ and $A R P E=2.5484$ ). The above results are showed in Table 2 .

\section{Empirical results of NGBM $(1,1)$ with ANN error correction}

Since forecast error can't be avoided, the researcher can try to estimate the forecast error in advance and then correct them on original estimated value. The process of obtaining the forecasting value by $\operatorname{NGBM}(1,1)$ with ANN Error Correction is described in the following.

(1) Denote the original forecasting error series, $E^{(0)}(1,7)=\{0,467.12, \ldots, 44757.57\}$,

(2) Calculate the standardized error $\varepsilon^{(0)}(k)$ by eq.(20).

(3) The modeling period is from 2004 to 2010. Use $E_{S}^{(0)}(k, k+3)$ to forecast $\varepsilon^{(0)}(k+4)$ by BP ANN. Therefore, there are three error series used for BP ANN. The parameters for computer program of Mat lab are

LP.lr = 0.1;

net.trainParam.epochs $=15000$;

net.trainParam.goal $=0.025$;

(4) The testing year is 2011 and the estimated GDP is 438502.02. The above results are showed in Table 2.

(5) For forecasting GDP of 2012, the new forecasting error series is $E_{S}^{(0)}(1,8)$. After repeating above procedure, the estimated value of GDP is 461534.45 . The above results are showed in Table 2.

\section{Comparisons of forecasting performance}

The error measures used for the forecasting comparison are the ARPE for in-sample data (2008-2010) and for out-of sample data (2011). Form Table 1, the RPE of NGBM $(1,1)$ from 2008 to 2010 are 0.0106 , -0.0723 and 0.0386 ; the RPE of NGBM $(1,1)$ with error correction from 2008 to 2010 are, $0.0118,0.0225$ and 0.0075 . Thus the former ARPE is. 0.0405 ; the latter $A R P E$ is 0.0139 . The model fitting results indicates $\operatorname{NGBM}(1,1)$ with error correction has lower forecast error.

Comparing the out-of sample data (2011), the RPE of NGBM $(1,1)$ is 0.0959 ; the $\mathrm{RPE}$ of $\operatorname{NGBM}(1,1)$ with error correction is 0.0608 . According to the results, the newly proposed model, NGBM $(1,1)$ with error correction, is with a highly forecasting accuracy.

To forecast the GDP of 2012, the data of 2011 have to inject into the grey forecasting system. As a results, the modeling error for NGBM (2008-2011) are $0.6826,-9.4583,-0.0942$ and 3.7822; the RPE of the proposed model (2008-2011) are, 0.0200, $-0.0415,0.0067$ and 0.0115 . Thus the former ARPE is. 3.5043; the latter ARPE is 0.0199. The model 
Citation: Chen Cl, Hsin PH (2016) Forecasting of Taiwan's Gross Domestic Product using the Novel Nonlinear Grey Bernoulli Model with ANN Error Correction. J Glob Econ 4: 177. doi:10.4172/2375-4389.1000177

Page 4 of 4

\begin{tabular}{|c|c|c|c|c|c|c|c|}
\hline \multirow[b]{2}{*}{ Year } & \multirow{2}{*}{$\begin{array}{l}\text { Actual value } \\
X^{0}(1,7)\end{array}$} & \multicolumn{3}{|c|}{ NGBM $\hat{X}^{0}(n=-0.06)$} & \multirow[b]{2}{*}{$\hat{\mathcal{E}}_{A N N}{ }^{(0)}$} & \multicolumn{2}{|c|}{ Proposed model } \\
\hline & & $\begin{array}{c}\text { Forecasted } \\
\text { values }\end{array}$ & $R P E$ & $\varepsilon^{(0)}$ & & $\begin{array}{c}\text { Forecasted } \\
\text { values }\end{array}$ & $R P E^{\star}$ \\
\hline 2004 & 339973 & 339973.00 & 0.0000 & 0.6217 & & & \\
\hline 2005 & 364832 & 364364.88 & 0.0013 & 0.6323 & & & \\
\hline 2006 & 376375 & 376349.30 & 0.0001 & 0.6222 & & & \\
\hline 2007 & 393134 & 386512.99 & 0.0168 & 0.7725 & & & \\
\hline 2008 & 400132 & 395877.79 & 0.0106 & 0.7186 & 0.6114 & 395427.02 & 0.0118 \\
\hline 2009 & 377529 & 404824.19 & -0.0723 & 0.0000 & 0.1934 & 386020.51 & -0.0225 \\
\hline 2010 & 430149 & 413537.71 & 0.0386 & 1.0000 & 0.9269 & 426939.44 & 0.0075 \\
\hline 2011 & 466881 & 422123.43 & 0.0959 & & 0.9947 & 438502.02 & 0.0608 \\
\hline
\end{tabular}

Note: ${ }^{*} R P E$ is relative percentage error as defined in eq(22); $\varepsilon^{(0)}$ is the standardized residual error, $\hat{\varepsilon}_{A N N}{ }^{(0)}$ the estimated standardized residual error by ANN.

Table 1: Actual value, forecasted value, and relative percentage error for Taiwan's GDP by NGBM $(1,1)$ and the proposed model (Units: US\$ million)

\begin{tabular}{|c|c|c|c|c|c|c|c|}
\hline \multirow[b]{2}{*}{ Year } & \multirow[b]{2}{*}{ Actual value $X^{0}(1,8)$} & \multicolumn{3}{|c|}{ NGBM $\hat{X}^{0}(n=-0.06)$} & \multirow[b]{2}{*}{$\hat{\varepsilon}_{A N N}^{(0)}$} & \multicolumn{2}{|c|}{ Proposed model } \\
\hline & & $\begin{array}{c}\text { Forecasted } \\
\text { values }\end{array}$ & $R P E$ & $\varepsilon^{(0)}$ & & $\begin{array}{c}\text { Forecasted } \\
\text { values }\end{array}$ & $R P E^{*}$ \\
\hline 2004 & 339973 & 339973.00 & 0.0000 & 0.3788 & & & \\
\hline 2005 & 364832 & 364529.18 & 0.0830 & 0.3853 & & & \\
\hline 2006 & 376375 & 371690.68 & 1.2446 & 0.3792 & & & \\
\hline 2007 & 393134 & 383328.73 & 2.4941 & 0.4707 & & & \\
\hline 2008 & 400132 & 397400.64 & 0.6826 & 0.4379 & 0.3268 & 392129.44 & 0.0200 \\
\hline 2009 & 377529 & 413236.91 & -9.4583 & 0.0000 & 0.2172 & 393178.85 & -0.0415 \\
\hline 2010 & 430149 & 430554.33 & -0.0942 & 0.6094 & 0.5694 & 427269.35 & 0.0067 \\
\hline 2011 & 466881 & 449222.68 & 3.7822 & 1.0000 & 0.9254 & 461505.86 & 0.0115 \\
\hline 2012 & & 469185.25 & & & 0.8075 & 461534.45 & 1 \\
\hline
\end{tabular}

Note: ${ }^{\star} R P E$ is relative percentage error as defined in eq $(22) ; \varepsilon{ }^{(0)}$ is the standardized residual error, $\hat{\varepsilon}_{A N N}{ }^{(0)}$ the estimated standardized residual error by ANN.

Table 2: Actual value, forecasted value, and relative percentage error for Taiwan's GDP by NGBM $(1,1)$ and the proposed model (Units: US\$ million)

fitting results also indicates the proposed model has lower forecast error.

The more accurate forecasting of future GDP trends is clearly of significant importance to both government and company financial directors. Both NGBM $(1,1)$ and the proposed model show that Taiwan is on the road to prosperity and away from financial meltdown.

\section{Conclusions}

Grey forecasting only requires a small data and make perfect in dealing with incomplete and uncertain information. Thus, grey forecasting is suitable for forecasting GDP. Among grey forecasting, $\operatorname{NGBM}(1,1)$ have been proven with high forecasting accuracy. This study proposes a hybrid grey forecasting, named NGBM $(1,1)$ with ANN error correction for Taiwan's GDP forecast. The idea of correcting forecast error in advance makes the proposed method useful in enhancing forecasting accuracy. In sample testing and out-of sample testing, the results support above contention.

\section{References}

1. Deng JL (1989) Introduction to Grey system. Journal of Grey System 1: 1-24.

2. Hsu Cl, Wen YH (1998) Improved grey prediction models for the trans-pacific air passenger market. Transportation Planning and Technology 22: 87-107.

3. Hsu CC, Chen CY (2003) Applications of improved grey prediction model for power demand forecasting. Energy Conversion and Management 44: 22412249 .

4. Hsu LC (2003) Applying the Grey prediction model to the global integrated circuit industry. Technological Forecasting and Social Change 70: 563-574.
5. Zhou P, Ang BW, Poh KL (2006) A trigonometric grey prediction approach to forecasting electricity demand. Energy 31: 2839-2847.

6. Jing $Y$ (2007) Application of residual grey model $(1,1)$ to the prediction of tuberculosis prevalence. Journal of Chongqing Medical University 3: 014.

7. Chen $\mathrm{Cl}$, Chen $\mathrm{HL}$, Chen SP (2008) Forecasting of foreign exchange rates of Taiwan's major trading partners by novel nonlinear Grey Bernoulli mode NGBM(1,1). Communications in Nonlinear Science and Numerical Simulation 13: $1194-1204$

8. Chen $\mathrm{Cl}$, Hsin PH, Wu CS (2010) Forecasting Taiwan's major stock indices by the Nash nonlinear grey Bernoulli model. Expert Systems with Applications 37: 7557-7562.

9. Wang ZX (2013) An optimized Nash nonlinear grey Bernoulli model for forecasting the main economic indices of high technology enterprises in China. Computers \& Industrial Engineering 64: 780-787.

10. Zhang LP (2014) An optimized Nash nonlinear grey Bernoulli model based on particle swarm optimization and its application in prediction for the incidence of Hepatitis B in Xinjiang, China. Computers in Biology and Medicine 49: 67-73.

11. Zill DG, Cullen MR (2000) Advanced Engineering Mathematics. Jones and Bartlett, Massachusetts.

12. Wen KL (2004) Grey Systems: Modeling and Prediction. Yang's , Arizona.

13. Ministry of Economic Affairs in Taiwan. 